\title{
Meta
}

Journal des traducteurs

Translators' Journal

\section{PEETERS, J. (2005) : La traduction : de la théorie à la pratique et retour, Rennes, Presses universitaires de Rennes, 168 p.}

\section{Hasnaa Kadiri}

Volume 53, numéro 4, décembre 2008

URI : https://id.erudit.org/iderudit/019658ar

DOI : https://doi.org/10.7202/019658ar

Aller au sommaire du numéro

Éditeur(s)

Les Presses de l'Université de Montréal

ISSN

0026-0452 (imprimé)

1492-1421 (numérique)

Découvrir la revue

Citer ce compte rendu

Kadiri, H. (2008). Compte rendu de [PEETERS, J. (2005) : La traduction : de la

théorie à la pratique et retour, Rennes, Presses universitaires de Rennes, 168 p.]

Meta, 53(4), 921-923. https://doi.org/10.7202/019658ar

Ce document est protégé par la loi sur le droit d'auteur. L'utilisation des services d'Érudit (y compris la reproduction) est assujettie à sa politique d'utilisation que vous pouvez consulter en ligne.

https://apropos.erudit.org/fr/usagers/politique-dutilisation/
Cet article est diffusé et préservé par Érudit.

Érudit est un consortium interuniversitaire sans but lucratif composé de l’Université de Montréal, l'Université Laval et l'Université du Québec à Montréal. Il a pour mission la promotion et la valorisation de la recherche. https://www.erudit.org/fr/ 
Peeters, J. (2005) : La traduction: de la théorie à la pratique et retour, Rennes, Presses universitaires de Rennes, 168 p.

Traduction, translation, transfert, transcription, transcodage et transposition signifient l'entrée en transe du traducteur dans l'univers des langues, souvent «asymétriques» (Venuti 1998). Le traducteur naviguant dans un «espace babélien» (Berman 1985), jongle avec les mots, les phrases, les références, les connotations, etc., à la recherche de la meilleure solution. Dans sa quête d'acceptabilité ou d'adéquation (Toury 1995), il prend ses décisions en fonction de telle théorie ou de telle autre; des théories foisonnantes, de la linguistique à l'herméneutique en passant par la sociolinguistique, le cognitivisme, le fonctionnalisme, etc. Louvrage La traduction: de la théorie à la pratique et retour regroupe quatorze articles illustrant, notamment, les interventions délibérées de traducteurs (Bastin 2007) et provenant d'horizons de réflexion assez différents. Il met en évidence le lien existant entre théorie et pratique et vice-versa dans l'activité de traduction. Chaque article examine la traduction sous un angle précis (théâtre, poésie, édition, etc.) et l'ensemble permet, sans redondance, une complémentarité des approches traductologiques.

Léona Van Vaerenbergh, de la Hogeschool Antwerpen, en Belgique, aborde trois sujets-clés: l'interdépendance de la linguistique et de la traductologie, car la recherche en traductologie contribue à la recherche linguistique, nous dit-elle; la description de la fonction du formateur, qui se trouve à mi-chemin entre celle du chercheur et celle du traducteur; et l'intérêt de la formation théorique du traducteur. En effet, elle affirme que tout traducteur doit disposer d'un bagage théorique et d'une compétence scientifique pour pouvoir - quand se présentent des problèmes de traduction ou une nouvelle situation de traduction - élaborer une solution adéquate au problème. Quant à David ar Rouz, de l'Université de HauteBretagne, il part de sa découverte du dialogue possible entre la traductologie et sa propre pratique de la traduction pour «établir un parallèle direct entre la pratique de l'autotraduction et [celle] de la traduction». L'auteur constate que toute analyse de la pratique mène à l'élaboration d'une théorie qui permet au traducteur de construire un discours sur la traduction.

Kerstin Jonasson, de l'Université Uppsala en Suède, montre que la théorie interprétative se distance clairement de la linguistique cognitive, bien que la construction même du sens se fasse grâce au «bagage cognitif» et au «contexte cognitif». Dans le sillage de Van Vaerenbergh, Yvon Keromnes, de l'Université de Metz, examine la relation asymétrique entre linguistique et traduction. Grâce à des exemples de traductions allemande(s), anglaise(s) et française(s), il démontre que c'est dans «l'étude de la traduction en contexte, [...] que la linguistique peut constituer un apport à la pratique de la traduction». Et Françoise Hammer, de l'Université Heidelberg en Allemagne, traite, sous l'angle de l'analyse textuelle et de la linguistique contrastive, de la formule juratoire pour montrer qu'elle constitue une provocation, non seulement sociale mais aussi linguistique.

À partir de quatre traductions, Paola Montera, de l'Université Lumière à Lyon, mesure au moyen de tests statistiques la fidélité dans soixante-quatorze syntagmes du roman The Waves de Virginia Woolf. Elle conclut que la notion de "fidélité est une approche descriptive et quantifiable «[...] qui reste en soi prescriptive». Gina Abou Fadel, de l'Université SaintJoseph au Liban, définit le texte comme une Imara (en arabe, terme générique qui englobe toute construction, le contraire d'une ruine) «qui est construite par un auteur» et où «le traducteur [...] est un locataire qui investit le texte source pour mieux le connaître sans pour autant en devenir propriétaire». Dans le texte sens et forme s'associent dans les textes «dans une relation étroite d'osmose, et [...] la forme permet [...] d'aboutir au sens».

De l'Université Catholique de l'Ouest, à Angers, Daniel Lévêque révèle la tâche complexe du traducteur face aux "actes délibérés» et aux «actes manqués» de l'auteur du texte littéraire, consistant à rendre ce que l'auteur a dit, ce qu'il a voulu dire et ce qu'il n'a pas 
voulu dire mais a quand même dit. Salama Ranomenjanahary Ramana, de l'Université d'Antananarivo (Madagascar), donne des exemples de traduction de métaphores du français au malgache où la difficulté, souligne-t-elle, est liée au fait que certains concepts sont absents en langue d'arrivée ou n'ont pas les mêmes « coordonnées culturelles » dans les deux langues en présence. Lalbila Yoda, de l'Université de Ouagadougou (Burkina Faso) et de l'Université de Groningen (Pays-Bas), fait le même constat avec la combinaison de langues français-bisa (langue minoritaire du Burkina Faso) en traduction médicale. Elle montre que, si les facteurs extratextuels sont identiques dans les deux langues (même skopos dans les textes source et cible), les facteurs intratextuels (linguistique et culturel) diffèrent d'une langue à l'autre.

Jean-Marc Gouanvic, de l'Université Concordia au Canada, se propose d'étudier le décentrement (ou scotomisation) traductif afin de définir les déterminations sociologiques de la pratique de la traduction. Il constate que le décentrement est intimement lié à la transformation que la traduction fait subir au texte de départ et qui se fait en fonction du champ littéraire cible. Il ajoute que la traduction n'est pas un simple transfert de sens d'une langueculture à une autre ni une translation des œuvres au-delà des frontières linguistiques nationales, car toute œuvre est le produit des habitus (Bourdieu). Il appuie sa réflexion sur l'analyse des traductions de plusieurs œuvres américaines, notamment celles de James Fenimore Cooper, de Mark Twain, de Jack London et d'Edgar Rice Burroughs. Alexandra Fukari, de l'Université de Graz (Autriche), s'interroge sur le rôle que jouent les maisons d'édition dans la production des traductions littéraires. En se basant sur le concept de patronage (Lefevere), elle explique la pratique de la sélection des textes traduits dans une société donnée. Dans la même veine, de l'Université de Paris III, Mila Dragovic-Drouet propose d'inscrire la traduction dans la chaîne éditoriale. Le dernier article, de Teresa Tomaszkiewicz de l'Université Adam Mickiewicz (Pologne), aborde la traduction intersémiotique (Jakobson, 1963) qui repose sur les mêmes principes que la traduction interlinguale.

Pouvons-nous en conclure que le lien entre théorie et pratique est saisissable? Les articles de ce collectif, réunis par Peeters, l'expliquent clairement. Pour ar Rouz, le lien est évident, la théorie n'est nulle autre que le fruit de la réflexion des chercheurs sur leurs pratiques. De la pratique de la traduction est née la théorie, et dans la théorie, le formateur puise ses méthodes d'enseignement. Aubin (2003) affirme «que si la théorie n'existait pas, il faudrait l'inventer, car aucun programme de formation ne serait viable sans une réflexion théorique sur la pratique du métier». Van Vaerenbergh explique que le formateur est à michemin entre le chercheur et le traducteur (et que, généralement, il endosse la responsabilité des trois en même temps). Il s'inspire des théories de la traduction pour «mener le traducteur à un niveau d'expert» (p. 24). Ce dernier sera muni des outils métalinguistiques et conceptuels nécessaires pour mener à bon port son entreprise de traduction, discuter avec son donneur d'ouvrage et le convaincre de la justesse de ses choix. Le schéma proposé par ar Rouz résume les relations existantes entre chercheur, traducteur et client:

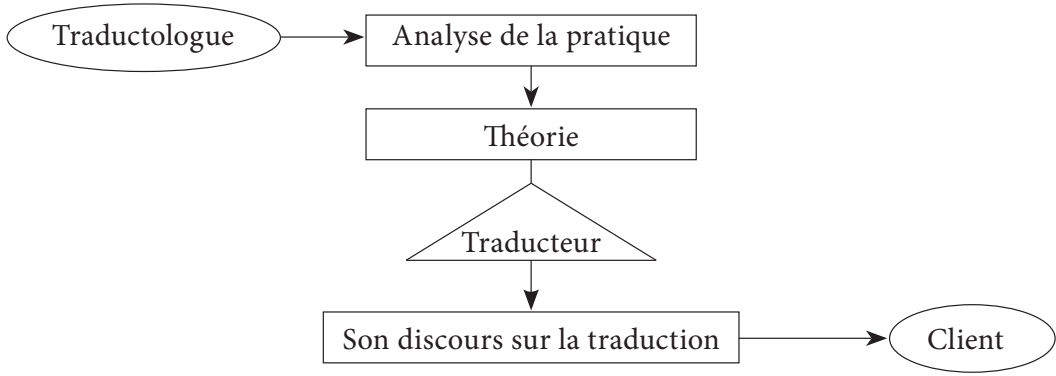

Soulignons aussi l'intérêt de se pencher sur les difficultés de traduire de ou vers des langues minoritaires (cas du malgache et du bisa). Les difficultés ne sont ni nouvelles ni 
étrangères au traducteur; elles exigent de lui une connaissance approfondie des langues et des cultures, mais aussi une ingéniosité et une créativité parfois hors du commun.

En compilant ces quatorze articles, Peeters a réussi à montrer l'intérêt porté par les chercheurs, issus d'horizons de réflexion différents, pour la théorie de la traduction et pour la place qu'elle occupe dans leur pratique traduisante. En fait, il poursuit un débat déjà amorcé en 2003 par d'autres chercheurs en traduction (voir Meta 48-3). Un débat passionnant et qui n'est certainement pas terminé!

HASNAA KADIRI

Université de Montréal, Montréal, Canada

\section{RÉFÉRENCES}

Aubin, M.-C. (2003) : «L'enseignement théorique dans un programme de formation universitaire en traduction", Meta 48-3, pp. 438-445.

Bastin, G. L. (2007): «Histoire, traductions et traductologie», in WотJAK, G. (dir.), Quo vadis Translatologie? Ein halbes Jahrhundert universitäre Ausbildung von Dolmetschern und Übersetzern in Leipzig, Berlin, Frank et Timme GmbH, pp. 35-44.

Berman, A. (1985): La traduction et la lettre ou l'auberge du lointain, Mauvezin, Trans-EuropRepress.

Toury, G. (1995) : Descriptive Translation Studies - And Beyond, Amsterdam/Philadelphia, John Benjamins.

Venuti, L. (1998): The Scandals of Translation: Towards an Ethics of Difference, London et New York, Routdlege.

Trandem, B. (2005): Discours sur le vif. Étude du processus de traduction des animismes du français en norvégien (Acta Humaniora 219), Oslo, Université d'Oslo, Faculté des lettres et sciences humaines, 298 p.

L'ouvrage présenté est une thèse de doctorat dont l'objet est une recherche empirique sur la traduction. Huit répondants (cinq traducteurs professionnels et trois non-professionnels) ont été invités à traduire deux textes publicitaires du français en norvégien, en pensant à haute voix. L'objectif principal de l'auteur est d'examiner le traitement des animismes, définis comme "représentation langagière qui consiste à attribuer à des choses inanimées ou à des entités abstraites des comportements, des sentiments ou des attitudes typiques des êtres vivants, quelle que soit la relation syntaxique entre ses constituants» (p. 120). Exemple: «Les vignes du Beaujolais voient courir leurs coteaux à l'ouest de la vallée de la Saône, entre Lyon et Mâcon.» Dans un deuxième temps, l'auteur souhaite contribuer à la mise en place d'une méthodologie expérimentale combinant deux méthodes de récolte de données: la réflexion parlée (le thinking-aloud) et Translog, logiciel qui enregistre l'activité du clavier de l'ordinateur. La problématique et les hypothèses sont énoncées au Chapitre 1. La méthode est présentée dans le Chapitre 2. Les Chapitres 3 et 4 fournissent le cadre théorique de la thèse. Le Chapitre 5 offre une vue d'ensemble du matériau, du profil des répondants et des conditions d'observation. Les Chapitres 6, 7 et 8 sont consacrés aux résultats. L'auteur y fournit d'abord des données quantitatives sur la performance globale des répondants (p. ex. la rapidité avec laquelle ils s'acquittent des tâches de traduction ou leur productivité, définie en termes du nombre de touches productrices de texte activées par minute). Elle passe ensuite à celles concernant plus spécifiquement le traitement des animismes, d'abord du point de vue du produit, puis de celui du processus de traduction. Le Chapitre 9 contient les conclusions générales ainsi que des propositions pour de nouvelles pistes de recherche. Suivent les références et les annexes, principalement sous forme des fichiers Translog et des traductions écrites. 